\title{
Zika: Probabilidad de establecimiento de su vector, Aedes aegypti, en Chile
}

\author{
Alberto J. Alaniz, Antonella Bacigalupo y Pedro E. Cattan
}

\section{Zika: Probability of establishment of its vector, Aedes aegypti, in Chile}

The Zika virus has raised world alarm in recent years, representing a major public health problem. In this study we evaluated the potential risk of exposure to Zika virus in Chile, associated with the probability of establishment of the vector Aedes aegypti in the country. Niche modelling techniques were used to project the bioclimatic requirements of the vector (global niches), identifying zones of high suitability for the species within the country. Then, the potential distribution of the vector in Chile was overlapped with the human population density, estimating the risk associated to the potential co-occurrence of both in a spatially explicit manner. We identified bioclimatic suitability for $A$. aegypti in continental Chile, from the northern tropical area to temperate regions, mainly in coastal zones. The exposed population could reach 1.8 million people, with 1.3 million in a medium level of potential risk and 21,000 in a high level. These results support that there is a significant probability of success for the Zika virus main vector to colonize continental Chile in case of an introduction. Therefore, prevention, monitoring, and control play an important role in avoiding the arrival of this vector to our country.

Key words: Zika virus, Aedes aegypti, habitat suitability, exposure risk, infectious vector diseases.

Palabras clave: Virus Zika, Aedes aegypti, idoneidad de hábitat, riesgo de exposición, enfermedades infecciosas vectoriales.

\section{Introducción}

$\mathrm{E}$ 1 virus Zika forma parte de la familia Flaviviridae, junto con otros virus que causan enfermedades infecciosas, tales como el dengue, la fiebre amarilla y el chikungunya ${ }^{1,2}$. Estos son dispersados principalmente a través de mosquitos pertenecientes al género Aedes, donde Aedes aegypti es considerado el vector principal de $\mathrm{Zika}^{2}$. Estos virus se caracterizan por generar distintos cuadros de complicación en pacientes adultos dependiendo de la cepa inoculada; sin embargo, Zika se caracteriza por afectar en forma permanente a fetos, los que son infectados a través de trasmisión transplacentaria ${ }^{3,4}$. Durante el año 2016 se reportaron en Colombia y Brasil numerosos casos de microcefalia, asociados a infecciones por Zika de las madres durante el verano de $2015-2016^{5,7}$. Por ello, el Zika es considerado un virus peligroso para la salud pública y de muy difícil control por las características reproductivas del insecto, especialmente en zonas tropicales, donde las altas temperaturas y humedad lo benefician ${ }^{8,9}$. A nivel global, 2.261 millones de personas estarían en niveles altos y muy altos de exposición, con algunos países presentando sobre el $90 \%$ de su población en riesgo potencial ${ }^{9}$.

En Chile continental, $A$. aegypti estuvo presente en la zona norte entre Arica y Caldera, pero se erradicó el año $1950^{10,11}$. Sin embargo, el reciente hallazgo de especímenes en la ciudad de Arica y los resultados de una reciente modelación epidemiológica ${ }^{19,12}$ sugieren que el riesgo es latente y se deben enfocar los esfuerzos en la prevención para mantener la erradicación del mosquito en el territorio continental. El presente estudio tiene por objetivo determinar la probabilidad potencial de establecimiento de $A$. aegypti en Chile, estimando la distribución potencial del insecto en el país, las zonas con riesgo de exposición y la población potencialmente expuesta.

\section{Método}

\section{Idoneidad de hábitat para el vector en Chile}

Se utilizó como metodología principal la modelación de nicho ecológico (MNE) basada en el algoritmo de máxima entropía, utilizando el software MaxEnt 3.4 ${ }^{13}$. Los MNE utilizan dos datos de entrada: localidades o presencias de la especie y variables ambientales, para estimar la idoneidad del hábitat para la especie ${ }^{14}$. Idoneidad puede ser entendida como el nivel de adecuación que posee una zona, dadas las características del nicho ecológico de la especie a modelar, y puede ser homologada a la abundancia potencial del organismo ${ }^{15,16}$. Se basa en proyectar los requerimientos de nicho ecológico de un organismo en un lugar determinado, mediante el principio de dualidad nicho-biotopo, permitiendo identificar la presencia de condiciones idóneas para el desarrollo de la especie en el lugar a colonizar ${ }^{17}$. Se recopilaron 20.203 presencias
Universidad de Chile, Santiago, Chile. Facultad de Ciencias Veterinarias y Pecuarias, Departamento de Ciencias Biológicas Animales, Laboratorio de Ecología de Ambientes Fragmentados (AJA). Laboratorio de Ecología ( $A B$, PEC).

Facultad de Ciencias Agronómicas, Departamento de Recursos Naturales Renovables, Laboratorio de Ecología de Ecosistemas (AJA). Centro de estudios en Ecología Espacial y Medio Ambiente - Ecogeografía, Santiago, Chile. (AJA)

Recibido: 1 de febrero de 2017 Aceptado: 19 de octubre de 2017

Correspondencia a:

Alberto J. Alaniz Baeza alberto.alaniz@ug.uchile.cl 


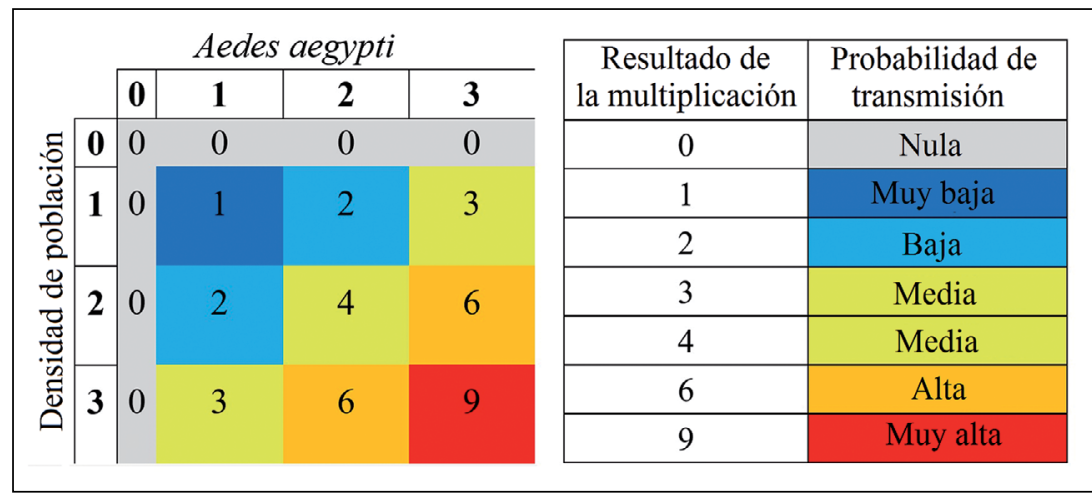

Figura 1. Esquema de multiplicación de las capas para estimar el riesgo.

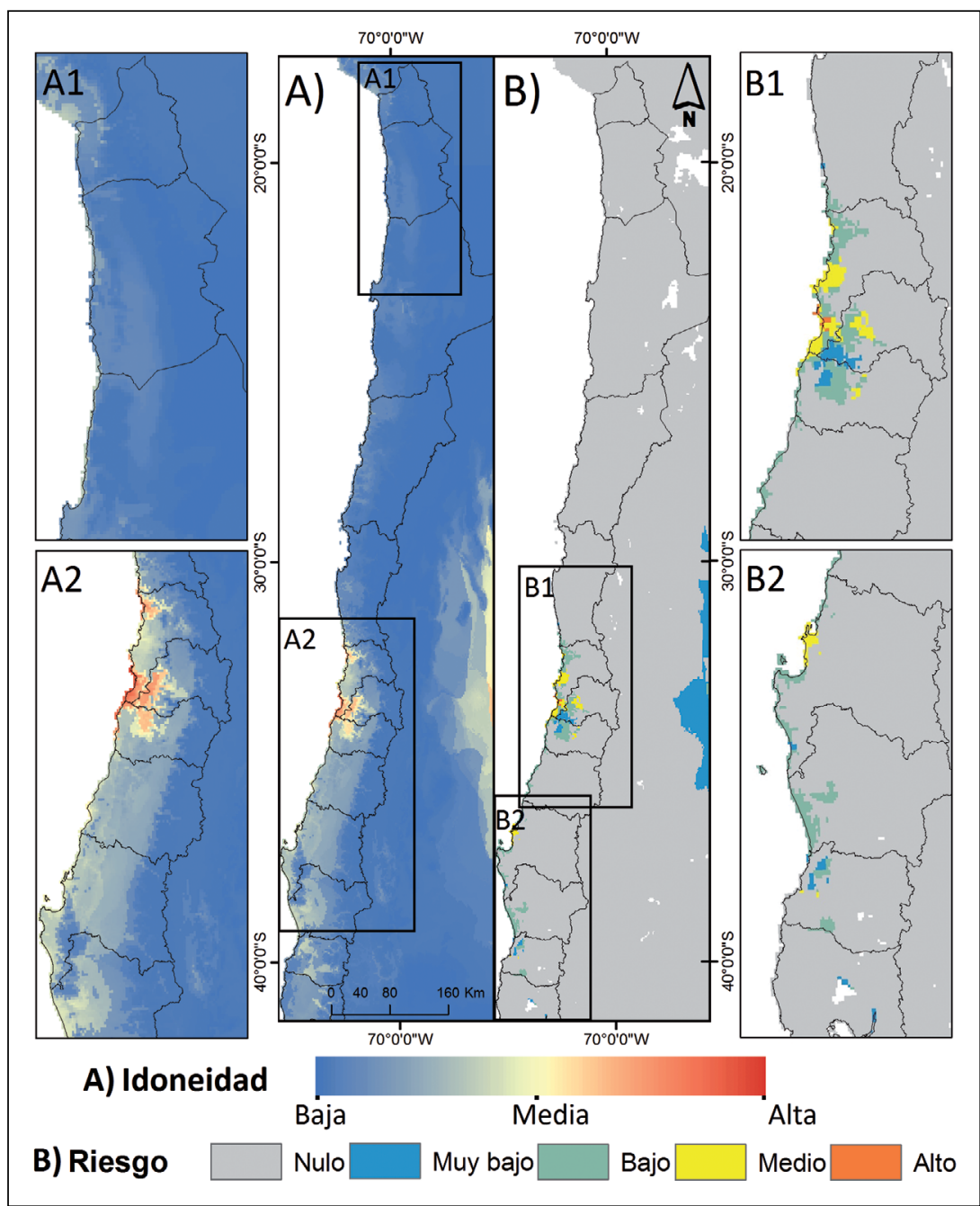

Figura 2. A: Mapa de idoneidad bioclimática de A. aegypti: A1) Extremo norte del país entre las Regiones de Arica y Parinacota y Antofagasta; A2) Centro-sur del país entre las regiones de Valparaíso y la Araucanía. B: Mapa de riesgo de exposición: B1) Zona entre las regiones de Coquimbo y el Maule; B2) Zona entre las Regiones de Biobío y Los Lagos. de $A$. aegypti para todo el mundo9. Como variables ambientales se utilizaron las 19 capas bioclimáticas del proyecto Worldclim ${ }^{18} \mathrm{y}$ la elevación, con una resolución espacial de $5 \times 5 \mathrm{~km}^{2}$ de pixel (Material suplementario 1). En primer lugar, se generó un modelo exploratorio con 15 réplicas y validación cruzada, en el cual se cuantifica la influencia de las variables ambientales (porcentaje de contribución e importancia de permutación). Para evitar el sobreajuste y la colinealidad de las variables, se evaluó el coeficiente de correlación absoluta entre variables expresado en correlogramas (Material suplementario 2) ${ }^{19}$. Posteriormente, se generó un modelo final con 100 réplicas utilizando el método de validación cruzada, con un intervalo de confianza de $95 \%$. Se utilizaron en este modelo final sólo las variables de mayor importancia para el modelo exploratorio y que tuviesen menos de $0,7 \mathrm{de}$ coeficiente de correlación absoluto. Todos los análisis estadísticos fueron realizados en el software R. Se analizó la idoneidad del hábitat para el vector en Chile como un símil a la probabilidad potencial de establecimiento, utilizando los resultados del modelo expresados en forma logística (probabilidades de 0 a 1 ).

\section{Estimación de la población en riesgo}

Se estimó el riesgo en forma espacialmente explícita, considerando como factores la abundancia potencial del vector (expresada en el MNE) y la densidad de población humana. Esto permite estimar el riesgo asociado a la probabilidad de co-ocurrencia del vector y del hospedero en un espacio geográfico determinado, considerando niveles de interacción esperada ${ }^{9}$. En primer lugar, se clasificó el MNE en cuatro niveles de probabilidad iguales asignándoles un valor numérico a cada uno (nulo $=0$, bajo $=1$, medio $=2$ y alto $=3$ ), transformando así la grilla continua del MNE en una grilla discreta de probabilidades, correspondiente a la capa de idoneidad discreta para A. aegypti. Se utilizó el mapa de densidad poblacional (habitantes $/ \mathrm{km}^{2}$ ) de SEDAC-NASA ${ }^{20}$, el que también fue clasificado en cuatro niveles de densidad poblacional discreta: nula $=0\left(0\right.$ a 1 habitantes $\left./ \mathrm{km}^{2}\right)$, baja $=1(1 \mathrm{a} 10$ habitantes $\left./ \mathrm{km}^{2}\right)$, media $=2\left(10\right.$ a 100 habitantes $\left./ \mathrm{km}^{2}\right)$ y alta $=3\left(>100\right.$ habitantes $\left./ \mathrm{km}^{2}\right)$. Posteriormente, los mapas clasificados fueron multiplicados siguiendo una matriz de doble entrada entre las capas de idoneidad discreta para A. aegypti y la densidad poblacional discreta en base a Alaniz y cols. ${ }^{9}$. Este proceso fue realizado utilizando la herramienta "Calculadora de raster" del Sistema de información Geográfica Qgis 2.8.2. El producto final consiste en un mapa del nivel de probabilidad de co-ocurrencia de vectores y población humana, desde nulo hasta muy alto, el que fue considerado como el riesgo potencial (Figura 1). Finalmente se cuantificó la población según nivel de riesgo utilizando un mapa de conteo de población por kilómetro cuadrado de la NASA ${ }^{21}$. 
Tabla 1. Niveles de riesgo de exposición a Aedes aegypti en Chile, en base a Alaniz et al. (in press)

\begin{tabular}{|c|c|c|c|c|c|c|c|c|}
\hline País & Muy alto & Alto & Medio & Bajo & Muy bajo & Total & Población total & $\%$ pop. en riesgo \\
\hline Chile & 0 & 21.830 & 1.320 .410 & 389.790 & 79.170 & 1.811 .200 & 18.131 .850 & $10.0 \%$ \\
\hline
\end{tabular}

\section{Resultados}

\section{Idoneidad de hábitat en Chile para el vector}

Las áreas con probabilidades de establecimiento para el insecto ocupan la zona costera desde Arica hasta la Región de Los Ríos. Por otro lado, se aprecia que el insecto tiene bajas posibilidades de establecerse en la zona interior de Chile debido a sus características bioclimáticas, decreciendo rápidamente sus probabilidades de éxito hacia el interior de continente (probabilidades $<0,05$ ) (Figura 2A). En la zona norte la probabilidad es baja pero presente (aproximadamente 0,10). En la zona central de Chile ésta aumenta considerablemente, hasta alcanzar su máximo en la Región de Valparaíso (aproximadamente 0,42).

\section{Estimación de la población en riesgo de exposición a Zika}

Las zonas con mayor riesgo para la población están representadas por las ciudades de Viña del Mar y Valparaíso (Figura 2B). En toda la zona costera del Norte Grande y Chico del país el nivel de exposición potencial es nulo, puesto que, si bien el mosquito tiene idoneidad de hábitat, ésta no es muy alta y no existen grandes densidades poblacionales que eleven el riesgo potencial de exposición.

La cantidad total de personas que tienen algún nivel de riesgo ascendería a 1.811 .200 , representando aproximadamente $10 \%$ de la población total de Chile. La mayor cantidad de población potencialmente expuesta (1,3 millones) se encontraría en zonas de nivel medio de riesgo de exposición, principalmente en las regiones de Valparaíso, Metropolitana, O'Higgins y Biobío (Figura 2B y Tabla 1).

\section{Discusión}

A diferencia de lo planteado por la $\mathrm{OMS}^{22}$, que señala que no existe posibilidad de ingreso y éxito del mosquito para Chile continental, los resultados arrojados por la presente modelación muestran lo contrario. Esto refuerza lo postulado por otros estudios que también evidenciaron idoneidad para el establecimiento de $A$. aegypti en Chile continental $^{9,23}$. Los lugares que poseen mayor idoneidad para el vector en general poseen influencia marítima de vaguada costera y nieblas bajas, lo cual podría permitir el establecimiento del mosquito, dada su necesidad de agua para reproducirse ${ }^{24}$. La baja probabilidad en zonas interiores podría estar asociada a la menor influencia costera en el control y variabilidad de temperatura, ya que estudios han identificado que el insecto tiene baja adecuación en zonas con mucha amplitud térmica ${ }^{9,25}$.

Respecto del riesgo para las personas, éste se presenta en una zona ampliamente poblada del territorio nacional, en la conurbación de Valparaíso-Viña del Mar, lo cual se vuelve bastante preocupante para la salud pública. Por otro lado, ha sido documentada la existencia de competencia para Zika de mosquitos del género Culex, los que están presentes en Chile ${ }^{26,27}$. Esto podría expandir la zona de riesgo asociado a la posible infección de nuevos vectores, por lo que es prioritario el control de $A$. aegypti en las fronteras del país, evitando así la introducción del virus. Otro factor que podría influir en la distribución del mosquito es el cambio climático, modificando así las predicciones del modelo presentado en este estudio ${ }^{28}$.

Se hace hincapié en el hecho de que este modelo constituye un escenario potencial, que se ha basado sólo en los requerimientos bioclimáticos de la especie. Sin embargo, la probabilidad de establecimiento podría estar influida por otros factores, como adaptaciones específicas, interacción con especies locales, condiciones locales de reproducción (disponibilidad de agua estancada para las larvas), capacidad de dispersión, entre otros ${ }^{28,29}$. Por lo anterior, el presente estudio constituye un antecedente que sugiere fuertemente que $A$. aegypti podría colonizar y establecerse en ciertos lugares de Chile continental. Las predicciones del modelo presentado aquí pueden considerarse conservadoras en términos de la distribución potencial predicha para el insecto, ya que, en general, las especies invasoras pueden ampliar su nicho ecológico en los lugares colonizados, colonizando zonas mayores a las predichas por la proyección ${ }^{17,30,31}$. En este sentido, recomendamos enfatizar las medidas de prevención, monitoreo y control del mosquito en las áreas definidas por el modelo.

\section{Resumen}

El virus Zika ha despertado una alarma mundial en los últimos años, representando un problema importante para la salud pública. En este estudio evaluamos el riesgo potencial de exposición a virus Zika en Chile, asociado a la probabilidad de establecimiento del vector Aedes aegypti en el país. Se utilizaron técnicas de modelación de nicho para proyectar los requerimientos bioclimáticos del vector (nicho global), identificando las zonas de alta idoneidad para la especie en el país. Luego, se superpuso la 
distribución potencial del vector en Chile con la densidad de población humana, estimando en forma espacialmente explícita el riesgo asociado a la co-ocurrencia potencial de ambos. Identificamos que existe idoneidad bioclimática para $A$. aegypti en Chile continental, desde el área tropical del norte hasta regiones templadas, principalmente en zonas costeras. La población potencialmente expuesta podría alcanzar 1,8 millones de personas, con 1,3 millo- nes en nivel medio y 21.000 en niveles altos de riesgo potencial de exposición. Los resultados expuestos aquí muestran que existe una significativa probabilidad de éxito de colonización del vector principal de virus Zika en Chile continental en caso de una introducción. Por lo tanto, la prevención, monitoreo y control se vuelven un tema importante para evitar la llegada de este vector a Chile continental.

\section{Referencias bibliográficas}

1.- Hayes E B. Zika virus outside Africa. Emerg. Infect Dis 2009; 15: 1347-50.

2.- $\quad$ Ayres C F J. Identification of Zika virus vectors and implications for control. Lancet Infect Dis 2016; 16 (3): 278-9.

3.- Besnard M, Lastère S, Teissier A, CaoLormeau V M, Musso D. Evidence of perinatal transmission of Zika virus, French Polynesia, December 2013 and February 2014. Euro Surveill 2014; 19 (13): pii $=20751$.

4.- Oliveira Melo A S, Malinger G, Ximenes R, Szejnfeld P O, Alves Sampaio S, Bispo De Filippis A M. Zika virus intrauterine infection causes fetal brain abnormality and microcephaly: Tip of the iceberg? Ultrasound Obstet Gynecol 2016; 47: 6-7.

5.- Cuevas E L, Tong V T, Rozo N, Valencia N, Pacheco O, Gilboa S M, et al. Preliminary report of microcephaly potentially associated with Zika virus infection during pregnancyColombia, January-November 2016. MMWR Morb Mortal Wkly Rep. 2016; 65: 1409-13.

6.- Collucci C. Colombia sees fourfold increase in microcephaly cases in a year. Br Med J 2016; 355: i6716.

7.- Magalhães-Barbosa MC, Prata-Barbosa A, Robaina JR, Raymundo CE, Lima-Setta F, Cunha AJ. Trends of the microcephaly and Zika virus outbreak in Brazil, January-July 2016. Travel Med Infect Dis 2016;14 (5): 458-63.

8.- Vorou R. Zika virus, vectors, reservoirs, amplifying hosts, and their potential to spread worldwide: what we know and what we should investigate urgently. Int J Infect Dis 2016; 48: 85-90.

9.- Alaniz A J, Bacigalupo A, Cattan P E. Spatial quantification of the world population potentially exposed to Zika virus. Int $\mathrm{J}$ Epidemiol 2017; 46: 966-75.

10.- Laval E. ¿Hubo Dengue autóctono en Chile? Rev Chilena Infectol 2001; 1: 69-71.

11.- González C R, Henry A, Reyes C, Aylwin M P, Escobar D, Fernández J, et al. Reintroduction of the invasive mosquito Aedes aegypti (Linnaeus) (Diptera: Culicidae) in northern Chile. IDESIA (Chile) 2016; 34: 1-5.

12.- Instituto de Salud Pública de Chile. Resultados de diagnóstico y confirmación de Culícidos.
Chile, 2010-2016. Boletín 2016; 6 (4): 1-11. Disponible en: http://www.ispch.cl/sites/ default/files/BoletinCulicidos-04052016C.pdf (Consultado: 28 de enero de 2017).

13.- Phillips S J, Anderson R P, DudÍk M, Schapire R E, Blair M E. Opening the black box: an open-source release of Maxent. Ecography (Cop) 2017; 40: 887-93.

14.- Elith J, Phillips S J, Hastie T, Dudík M, Chee Y E, Yates C J. A statistical explanation of MaxEnt for ecologists. Divers Distrib 2011; 17: 43-57.

15.- Pike D A. Climate influences the global distribution of sea turtle nesting. Glob Ecol Biogeogr 2013; 22: 555-66.

16.- VanDerWal J, Shoo L P, Johnson C N, Williams S E. Abundance and the environmental niche: environmental suitability estimated from niche models predicts the upper limit of local abundance. Am Nat 2009; 174: 282-91.

17.- Gallien L, Douzet R, Pratte S, Zimmermann NE, Thuiller W. Invasive species distribution models-how violating the equilibrium assumption can create new insights. Glob Ecol Biogeogr 2012; 21: 1126-36.

18.- Hijmans R J, Cameron S E, Parra J L, Jones P G, Jarvis A. Very high resolution interpolated climate surfaces for global land areas. Int $\mathrm{J}$ Climatol. 2005; 25 (15): 1965-78.

19.- Bradley C. The absolute correlation coefficient. Math Gazette 1985; 69 (447): 12-7.

20.- Center for International Earth Science Information Network (CIESIN), Columbia University and Centro Internacional de Agricultura Tropical (CIAT). Gridded Population of the World Version 3 (GPWv3): Population Density Grids. Palisades, NY: Socioeconomic Data and Applications Center (SEDAC), Columbia University.

21.- Center for International Earth Science Information Network (CIESIN), Columbia University; International Food Policy Research Institute (IFPRI); the World Bank; and Centro Internacional de Agricultura Tropical (CIAT). Global Rural-Urban Mapping Project, Version 1 (GRUMPv1): Population Count Grid. Palisades, NY: Socioeconomic Data and Applications Center (SEDAC), Columbia University.
22.- Organización Mundial de la Salud. Zika virus. Retrieved February 05, 2016, Disponible en: http://www.who.int/mediacentre/factsheets/ zika/en/ (Revisado: 28 de enero de 2017).

23.- Kraemer M U G, Sinka M, Duda K A, Mylne A Q N, Shearer F M, Barker C M, et al. The global distribution of the arbovirus vectors Aedes aegypti and Ae. albopictus. eLife 2015; 4: 1-18.

24.- Ramasamy R, Surendran S N, Jude P J, Dharshini S, Vinobaba M. Larval development of Aedes aegypti and Aedes albopictus in periurban brackish water and its implications for transmission of arboviral diseases. PLoS Negl Trop Dis. 2011; 5 (11).

25.- Carrington L B, Armijos M V, Lambrechts L, Baker C M, Scott T W. Effects of fluctuating daily temperatures at critical thermal extremes on Aedes aegypti life-history traits. PLoSOne 2013; 8 (3): e58824.

26.- Huang Y-J S, Ayers V B, Lyons A C, Unlu I, Alto B W, Cohnstaedt L W, et al. Culex species mosquitoes and Zika virus. VectorBorne Zoonotic Dis 2016; 16 (10): 673. doi: 10.1089/vbz.2016.2058. Epub 2016 Aug 24.

27.- Guo X, Li C, Deng Y, Xing D, Liu Q M, Wu $\mathrm{Q}$, et al. Culex pipiens quinquefasciatus: a potential vector to transmit Zika virus. Emerg Microbes Infect 2016; 5 (9): e102.

28.- Campbell-Lendrum D, Manga L, Bagayoko M, Sommerfeld J. Climate change and vectorborne diseases: what are the implications for public health research and policy? Philos Trans R Soc 2015; 370: 20130552.

29.- Verdonschot P F M, Besse-Lototskaya A A. Flight distance of mosquitoes (Culicidae): A metadata analysis to support the management of barrier zones around rewetted and newly constructed wetlands. Limnologica 2014; 45 : 69-79.

30.- Veran S, Piry S, Ternois V, Meynard C N, Facon B, Estoup A. Modeling spatial expansion of invasive alien species: Relative contributions of environmental and anthropogenic factors to the spreading of the harlequin ladybird in France. Ecography (Cop) 2016; 39: 665-75.

31.- Hill M P, Gallardo B, Terblanche J S. A global assessment of climatic niche shifts and human influence in insect invasions. Glob Ecol Biogeogr 2017; 26: 679-89. 